\title{
A Cooperation-Aware Routing Scheme for Fast Varying Fading Wireless Channels
}

\author{
Daniel de O. Cunha, Otto Carlos M. B. Duarte, and Guy Pujolle
}

\begin{abstract}
In this letter, we propose a cooperation-aware routing scheme based on long-term statistics of cooperative links. The proposed scheme fully exploits cooperation on fading scenarios and proves to be more efficient than greedy cooperation-aware routing based on instantaneous link conditions. Moreover, the performance improvement is even higher when cooperative and non-cooperative nodes coexist.
\end{abstract}

Index Terms-Routing, cooperative diversity, fading.

\section{INTRODUCTION}

$\mathbf{C}$ OOPERATIVE communication is a powerful technique to provide spatial diversity to single-antenna nodes. The cooperative protocols allow nodes to share their antennas and act as relays for communications of their neighbors. The resulting cooperative diversity mitigates the effect of fading, increasing the robustness of transmissions. A cooperationaware routing scheme is essential to fully exploit the benefits of cooperation on multi-hop networks.

Most of the work on cooperation-aware routing deals with minimum-energy routing on single rate cooperative networks. Khandani et al. derived lower bounds for energy savings obtained through cooperative routing on regular networks [1]. Yang et al. propose polynomial heuristics to find cooperative energy-saving routes [2]. Another approach is to search for cooperation-aware routes on multirate networks aiming to increase the achieved throughput [3]. In both schemes, the analyses and proposed algorithms assume static networks, i.e., networks where the channel variations are slow compared to the response time of the routing layer, allowing to optimize routes based on instantaneous network conditions, hereafter called greedy schemes. Nevertheless, measurements on wireless channels proved that whereas the average coherence time of fading channels linking static nodes is in the order of tens or hundreds of milliseconds [4]-[6], the average round-trip-time (RTT) of lightly loaded wireless networks is already in the order of hundreds or thousands of milliseconds [7]. Therefore, on these fast varying environments, greedy cooperative routes experience lower performance because routing is not able to adapt for each single channel variation. As a result, a route selected to optimize performance on a specific fading condition will still be used on different configurations. Depending on the stability of the links, the greedy route may present significant variations, reducing its long-term performance.

In this letter, we analyze cooperation-aware routing for fading networks and show that greedy algorithms are not adequate. Then, we propose a Weighted Cooperation-Aware

Daniel de O. Cunha is with the LIP6 laboratory of the University of Paris 6, France, and GTA/COPPE/POLI laboratory of the Federal University of Rio de Janeiro, Brazil (e-mail: daniel.cunha@lip6.fr).

Otto Carlos M. B. Duarte is with the GTA/COPPE/POLI laboratory of the Federal University of Rio de Janeiro, Brazil (e-mail: otto@gta.ufrj.br).

Guy Pujolle is with the LIP6 laboratory of the University of Paris 6, France (e-mail: guy.pujolle@lip6.fr). routing metric (WCA), which uses long term channel information to prioritize routes composed by stable links. The key idea is to find the route with the highest probability of presenting good conditions instead of finding the optimal route based on instantaneous link conditions. The analyses are conducted both on entirely cooperative networks and on hybrid networks, where cooperative and non-cooperative nodes coexist. The results show that greedy cooperation-aware algorithms do not fully exploit cooperation on typical fading networks. Furthermore, the proposed weighted cooperation-aware metric allows the selection of more stable routes, which present higher endto-end success probabilities. The improvement obtained by the proposed metric is even more significant on hybrid networks, where there are fewer cooperation opportunities.

\section{System Model}

We assume nodes employ the Non-orthogonal Amplify-andForward (NAF) protocol [8] at the physical layer and only one relay per cooperative transmission. If more than one relay is available, the Medium Access Control (MAC) layer selects the best one. Moreover, cooperation is used selectively, i.e., it is only used when the instantaneous capacity achieved by the cooperative configuration is higher than the capacity of the conventional non-cooperative link [9]. Thus, the instantaneous capacity of the cooperative link, $C^{\prime}$, is

$$
C^{\prime}=\max \left\{C\left(h_{S D}\right), C_{N A F}\left(R^{*}\right)\right\},
$$

where $C\left(h_{S D}\right)$ is the capacity of the source-destination direct channel in the non-cooperative configuration and $C_{N A F}\left(R^{*}\right)$ is the capacity of the cooperative configuration using the best available relay. All the channels are assumed to present Rayleigh fading because it is a worst-case condition.

The analyses are derived based on parameters of the OFDM mode of the IEEE $802.11 \mathrm{~g}$ standard. The minimum transmission rate considered is $6 \mathrm{Mbps}$, whereas the maximum rate is $54 \mathrm{Mbps}$. The outage capacity of a transmission rate is the channel capacity required to successfully employ the given transmission rate. Based on the coding rate and the number of bits per sub-carrier, we can derive the outage capacity for each rate. For the $6 \mathrm{Mbps}$ transmission rate, which uses one bit per sub-carrier and a coding rate of 0.5 , the outage capacity is 0.5 bits per channel use (bpcu). On the other hand, the $54 \mathrm{Mbps}$ transmission rate uses six bits per sub-carrier and a coding rate of 0.75 , which gives an outage capacity of $4.5 \mathrm{bpcu}$. These two outage capacities are called $C_{\min }$ and $C_{\max }$, respectively. Moreover, the packet transmission time, $T_{p}$, is modeled as

$$
T_{p}=\frac{K_{d}}{C}+K_{o}
$$

where $K_{d}$ is a constant related to the packet size, $K_{o}$ is a constant associated to the protocol overhead, and $C$ is the 
instantaneous channel capacity. Assuming 1024-byte packets and calculating the packet transmission time as in [10], $K_{d}$ and $K_{o}$ are set to $675 \mu s$ and $250 \mu s$, respectively.

\section{LIMITATIONS OF GREEDY SCHEMES}

Cooperation-aware routing is different from conventional routing with the use of opportunistic cooperation. On the first case, the routing metric is defined using information about the cooperative opportunities, e.g., the quality of available relays. On the other hand, opportunistic cooperation with conventional routing selects routes without considering cooperationrelated information and, therefore, there are no guarantees that cooperative opportunities are available. Although it is intuitive that cooperation-aware routing is more efficient than conventional routing with opportunistic cooperation, finding the best cooperation-aware policy for fast varying networks is not trivial.

Consider a simple scenario where a greedy routing algorithm must choose among three links based on their instantaneous capacities. Figure 1 shows the probability density of the available channel capacity of these three links when their direct source-destination channels $\left(h_{S D}\right)$ present an average SNR of $5 \mathrm{~dB}$. Whereas Link A is a non-cooperative link with no relay available, links $\mathrm{B}$ and $\mathrm{C}$ use cooperation and have, respectively, 1 and 2 available relays. We also assume that source-relay $\left(h_{S R}\right)$ and relay-destination $\left(h_{R D}\right)$ channels present an average SNR $10 \mathrm{~dB}$ higher than the direct source-destination channel. As the number of available relays increase, the cooperation opportunities also increase and the outage probability of the link decreases. We obtain the outage probability integrating the probability densities from 0 to $C_{m i n}$. According to Fig-

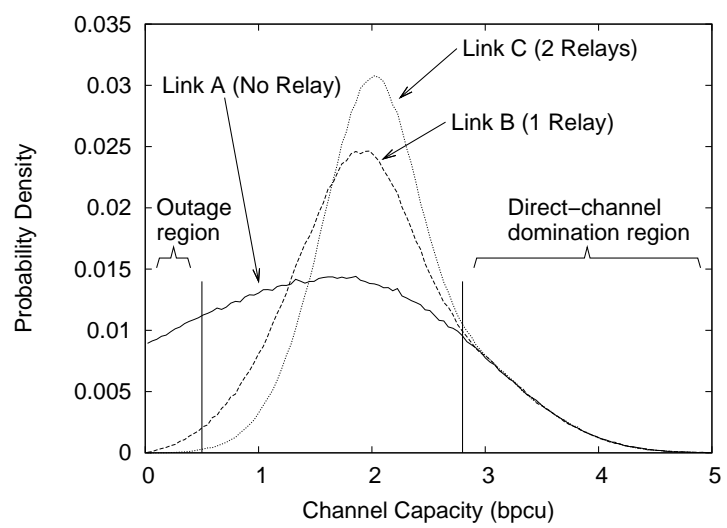

Figure 1. Probability densities of the analyzed channels capacities.

ure 1, Link A presents low instantaneous capacities with high probability. For the given $C_{m i n}=0.5$ (Section II), the outage probability of Link A is $12 \%$, of Link B is as low as 1\%, and of Link $\mathrm{C}$ is only $0.08 \%$. On the other hand, in the high-capacity region, the probability densities of the three links are similar. This is due to the nature of the cooperative protocol, which does not aim to increase the maximum capacity of the link, but to improve its worst-case condition. Usually, when a link has a high instantaneous capacity, it is the result of a good direct channel $\left(h_{S D}\right)$, in which case cooperation is not necessary. When a greedy cooperation-aware algorithm looks for the link with higher capacity it is in fact giving more importance to the cases where cooperation is not used.

The consequence of the use of greedy algorithms is clear when we observe the probability of a link being selected based on its instantaneous capacity as a function of the average SNR of its direct channel. Figure 2 shows the results. As stated before, the average SNR of $h_{S R}$ and $h_{R D}$ are $10 \mathrm{~dB}$ higher.

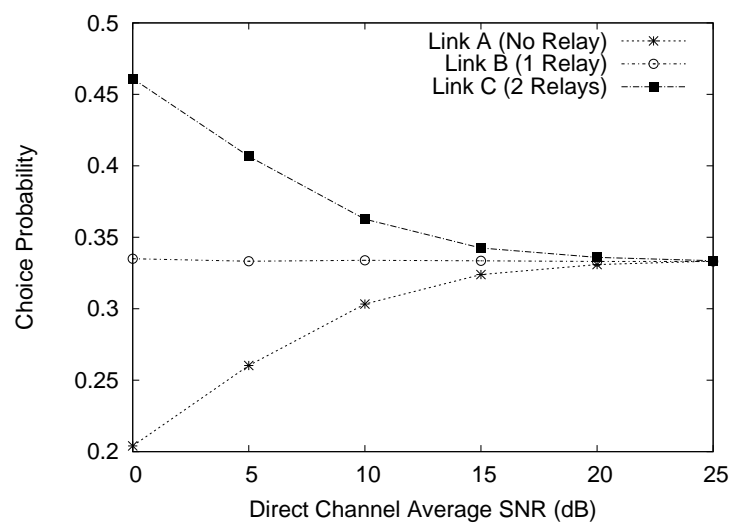

Figure 2. Choice probability of the different links based on instantaneous channel conditions.

A greedy routing algorithm frequently selects Link A, as shows Figure 2. Nevertheless, Link C is clearly the best choice because it presents a significantly lower outage probability. For the conditions analyzed on Figure 1, where the SNR on the direct channel is equal to $5 \mathrm{~dB}$, Link $\mathrm{A}$ is badly selected with a probability higher than 0.25 . Our proposal enhances cooperation-aware routing to avoid this situation.

\section{Weighted COOPERATION-AWARE ROUTING}

The greedy algorithms choose routes that have a low longterm efficiency due to fading because these algorithms look for optimizing routes considering instantaneous conditions. To couple with this problem, we propose the use of a cooperation-aware policy that takes into account long-term channel statistics. Thus, we propose a new routing metric, called Weighted Cooperation-Aware (WCA), which is based on the outage probability of the cooperative link, $P_{\text {out }}(l)$, and its average useful capacity, $E\left[C_{u}(l)\right]$. The outage probability of a cooperative link is the probability of its instantaneous capacity, $C^{\prime}$, getting lower than $C_{\min }$ and the useful capacity of the link is

$$
C_{u}(l)=\left\{\begin{array}{ll}
0, & C^{\prime}(l)<C_{\min } \\
C^{\prime}(l), & C_{\min } \leq C^{\prime}(l) \leq C_{\max } \\
C_{\max }, & C^{\prime}(l)>C_{\max }
\end{array} .\right.
$$

Equation (3) reflects that transmissions fail if the instantaneous capacity is lower than $C_{\min }$ and that the node cannot benefit from a capacity higher than $C_{\max }$. Therefore, to take the channel variations into account, we propose the Weighted Cooperation-Aware metric given by

$$
W C A(l)=\left(\frac{K_{d}}{E\left[C_{u}(l)\right]}+K_{o}\right) * \frac{1}{1-P_{\text {out }}(l)} .
$$

To analyze the improvement obtained by the proposed Weighted Cooperation-Aware (WCA) metric, we performed 
simulations and compared it to a Greedy Cooperation-Aware (GCA) metric and to Minimum number of Hops routing with Opportunistic Cooperation (MH-OC). For each run, we considered $10^{6}$ fading realizations. Whereas the Weighted Cooperation-Aware metric is calculated as in (4), the Greedy Cooperation-Aware metric is the transmission time through the link, as in (2), based on the initial network configuration. The three metrics are compared based on the end-to-end success probability of the selected routes.

The transmission range of the nodes, $r$, is set to $70 \mathrm{~m}$ and the transmitting power is configured to result in an outage probability of the direct channels equal to $50 \%$ at this distance. We use the log-distance large-scale attenuation model with $\beta=2.7$. The simulation area is a rectangle with sides $\pi r$ and $6 r$. The communication is started by a node at the coordinates $\left(\pi \frac{r}{2}, 1.5 r\right)$ and its sink is located at $\left(\pi \frac{r}{2}, 4.5 r\right)$. The position of these nodes is chosen to reduce the influence of the border effect on the selected routes. We vary the average number of nodes per coverage area to observe the effect of density on the results. We used Matlab to perform the simulations and 95\% confidence intervals are showed by vertical bars.

Figure 3 shows the results obtained for networks composed solely by cooperative nodes. We can observe that greedy cooperation-aware routing performs better than conventional routing with opportunistic cooperation. Moreover, the Weighted Cooperation-Aware metric presents the best results with an end-to-end success probability from 15 up to $19 \%$ higher when compared to the Greedy Cooperation-Aware metric.

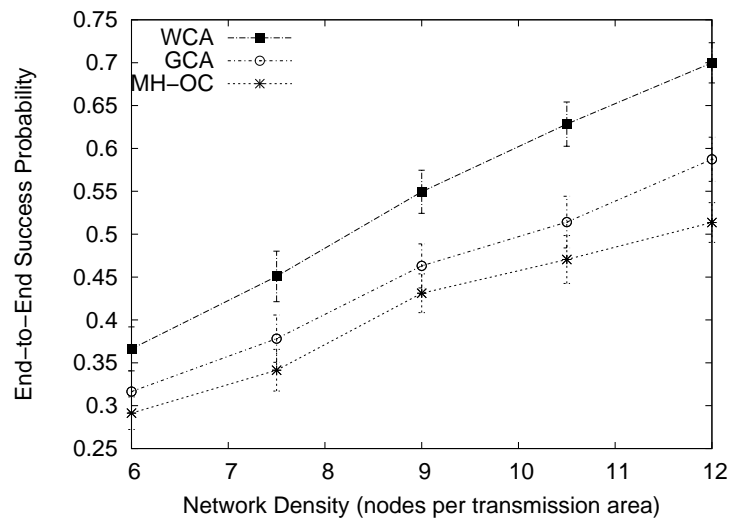

Figure 3. Success probability on a fully cooperative network.

Figure 4 shows the results obtained for an hybrid network where half the nodes does not implement cooperation. In this case, there are less cooperative links because both the source and the destination of a link must be cooperative to allow the use of cooperation. This scenario represents transitional networks, where legacy non-cooperative devices coexist with the cooperative ones. In this scenario, the overall success probability is lower. Moreover, the gain of the WCA metric over the GCA metric is higher. WCA results in end-to-end success probabilities up to $74 \%$ higher than the ones obtained by GCA. In fact, on hybrid networks where the cooperative opportunities are scarcer, GCA presents similar results to conventional routing with opportunistic cooperation (MH-OC).

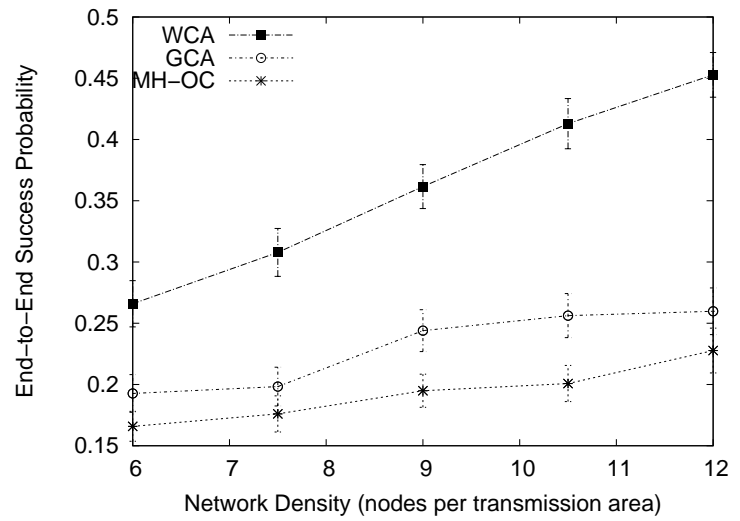

Figure 4. Success probability on an hybrid network.

\section{CONCLUSiOnS}

We addressed the problem of cooperation-aware routing on networks where the variations due to fading are fast in comparison to the response time of the routing. The analyses show that on this scenario greedy cooperation-aware algorithms fail to fully exploit the benefits of cooperation. We propose a new cooperation-aware routing metric, called Weighted Cooperation-Aware, which takes into account the long-term behavior of the links. The results show a significant improvement of the end-to-end success probability of communications. Finally, performance improvement obtained by the proposed metric is higher when cooperative nodes coexist with non-cooperative nodes, because there are less cooperative opportunities. These results suggest that the proposed metric is especially suitable for scenarios where cooperation is incrementally deployed.

\section{REFERENCES}

[1] A. E. Khandani, J. Abounadi, E. Modiano, and L. Zheng, "Cooperative routing in wireless networks," in Allerton Conference on Communications, Control and Computing, 2003.

[2] Z. Yang, J. Liu, and A. Høst-Madsen, "Cooperative routing and power allocation in ad-hoc networks," in IEEE Globecom, 2005.

[3] E. Beres and R. Adve, "Cooperation and routing in multi-hop networks," in IEEE International Conference on Communications, 2007.

[4] J. Moss, M. Fitton, A. M. Street, K. M. Brown, C. C. Constantinou, and D. J. Edwards, "Spatio-temporal variability analysis of the wideband microcellular environment," in IEEE Vehicular Technology Conference, 1998.

[5] H. MacLeod, C. Loadman, and Z. Chen, "Experimental studies of the 2.4-GHz ISM wireless indoor channel," in 3rd Annual Communication Networks and Services Research Conference (CNSR'05), 2005.

[6] R. P. Torres, B. Cobo, D. Mavares, F. Medina, S. Loredo, and M. Engels, "Measurement and statistical analysis of the temporal variations of a fixed wireless link at $3.5 \mathrm{GHz}$," Wireless Personal Communications, vol. 37, no. 1-2, Apr. 2006.

[7] M. E. M. Campista, D. G. Passos, P. M. Esposito, I. M. Moraes, C. V. N Albuquerque, D. C. M. Saade, M. G. Rubinstein, L. H. M. K. Costa, and O. C. M. B. Duarte, "Routing metrics and protocols for wireless mesh networks," IEEE Network, vol. 1, no. 22, Jan. 2008.

[8] R. Nabar, H. Bölcskei, and F. Kneubühler, "Fading relay channels: Performance limits and space-time signal design," IEEE Journal on Selected Areas of Communication, vol. 22, no. 6, Aug. 2004.

[9] J. Cai, X. Shen, J. W. Mark, and A. S. Alfa, "Semi-distributed user relaying algorithm for amplify-and-forward wireless relay networks," IEEE Transactions on Wireless Communications, vol. 7, no. 4, Apr. 2008.

[10] M.-J. Ho, J. Wang, K. Shelby, and H. Haisch, "IEEE 802.11g OFDM WLAN throughput performance," in IEEE VTC-Fall, 2003. 